\title{
Aerogel Antennas Communications Study Using Error Vector Magnitude Measurements
}

\author{
Félix A. Miranda*, Carl H. Mueller ${ }^{+}$, and Mary Ann B. Meador* \\ *National Aeronautics and Space Administration \\ Glenn Research Center, Cleveland, OH 44135, U.S.A \\ +QinetiQ-NA, Cleveland, OH 44135, U.S.A. \\ 2014 IEEE International Symposium on Antennas and Propagation \\ And USNC/URSI National Radio Science Meeting \\ July 6-11, 2014, Memphis, TN
}

Paper Number: 1853

Technical Session 338: Applications of Phased Arrays, Wednesday, July 09, 2014

Presentation: 338.3 


\section{Abstract}

This presentation discusses an aerogel antennas communication study using error vector magnitude (EVM) measurements. The study was performed using $2 \times 4$ element polyimide (PI) aerogel-based phased arrays designed for operation at $5 \mathrm{GHz}$ as transmit (Tx) and receive (Rx) antennas separated by a line of sight (LOS) distance of 8.5 meters. The results of the EVM measurements demonstrate that polyimide aerogel antennas work appropriately to support digital communication links with typically used modulation schemes such as QPSK and $\pi / 4$ DQPSK. As such, PI aerogel antennas with higher gain, larger bandwidth and lower mass than typically used microwave laminates could be suitable to enable aerospaceto- ground communication links with enough channel capacity to support voice, data and video links from CubeSats, unmanned air vehicles (UAV), and commercial aircraft. 


\section{Outline}

$>$ Background

$>$ Motivation

$>$ Experiments

- Antenna, Design, Fabrication and Performance

- Error Vector Magnitude Measurements

$>$ Results

$>$ Conclusions

$>$ Relevant References 


\section{Background}

$>$ Design and optimization of aerospace communication systems is of great interest in the aviation for government (e.g., NASA, Department of Defense, etc.) and the commercial sector.

A typical commercial and/or military aircraft could have as many as 15 to 100 antenna systems.

Large number of antennas adds weight, increases complexity, and poses structural integrity challenges. The latter is exacerbated in commuter and general aviation aircraft because of more limited space for antenna placement.

$>$ Approaches that could reduce the mass and/or the number of antennas for communication and/or radar in aircraft and any other pertinent airborne platform (e.g., long duration, high altitude platforms) without sacrificing performance are highly desired.

$>$ As highly porous solids possessing low density and low dielectric permittivity combined with good mechanical properties, polyimide (PI) aerogels offer great promise as an enabling technology for lightweight aircraft antenna systems.

$>$ While PI aerogels have been aggressively explored for thermal insulation, barely any effort has been made to leverage these materials for antennas.

$>$ With the support of NASA's Aeronautics Research Institute (NARI), our group has performed research, analysis, and proof-of-concept development of PI aerogel antennas for aerospace communications. 


\section{Motivation}

Evaluate potential for using polyimide (PI) aerogels as a substrate for antennas 


\section{What are Aerogels?}

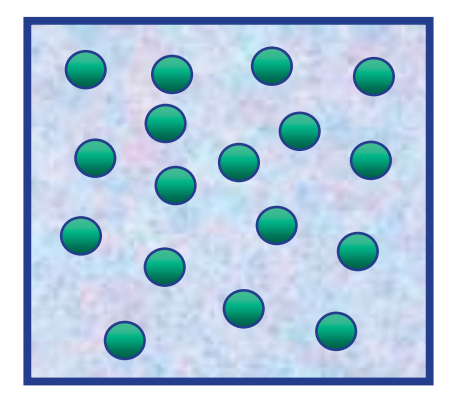

Sol

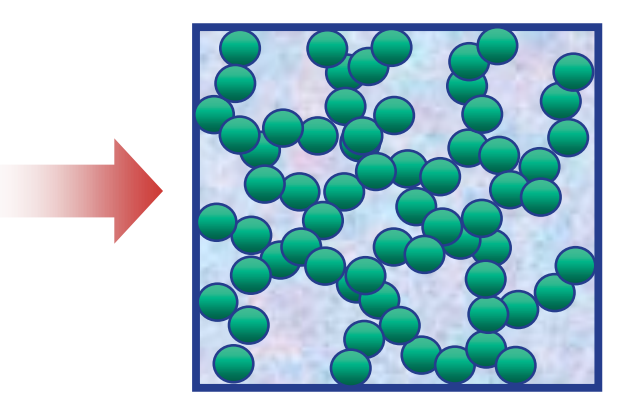

Gel

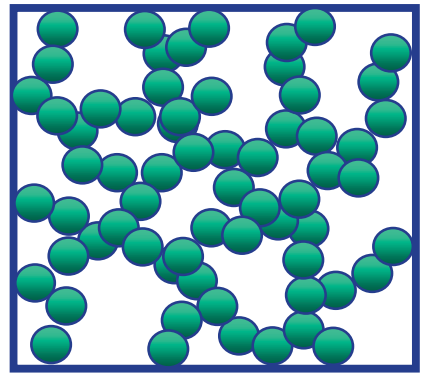

Aerogel

$>$ Highly porous solids made by drying a wet gel without shrinking

$>$ Pore sizes extremely small (typically $10-40 \mathrm{~nm}$ ) - very good insulation

$>$ High surface areas

$>$ Density as low as $0.008 \mathrm{~g} / \mathrm{cm}^{3}$

$>$ Low density $=$ low dielectric properties

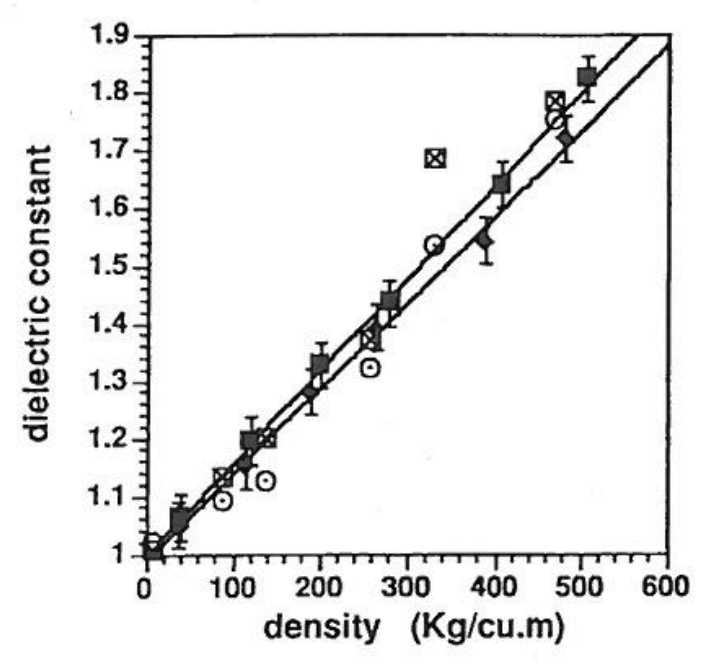

$2 \mathrm{GHz}$ (as prepared)

○ $10 \mathrm{GHz}$ (as prepared)

口 $18 \cdot 40 \mathrm{GHz}$ (as prepared)

- $18-40 \mathrm{GHz}$ (baked)

Dielectric constants of silica aerogels as a function of density 


\section{What are Aerogels? (cont.)}

$>$ Previously studied silica aerogels

- Many amazing properties, including low relative dielectric constant, low density

- However, very fragile

$>$ Recently developed polyimide aerogels

- Same low density

- Mechanically robust

$>$ Take advantage of the superior mechanical properties, light weight, low dielectric properties of polyimide aerogels to use as antenna substrate

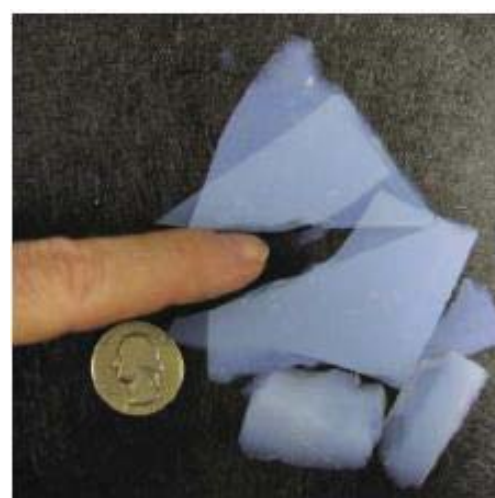

Silica aerogel

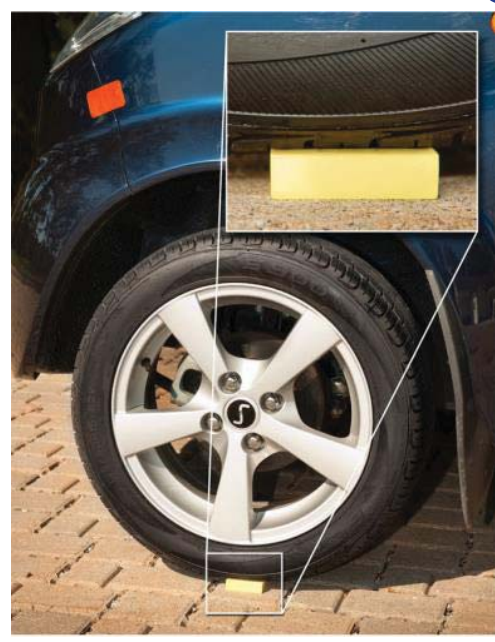

Polyimide aerogel 
Aerogel formulations with relative dielectric constants as low as 1.08 identified. Dielectric properties depend only on density
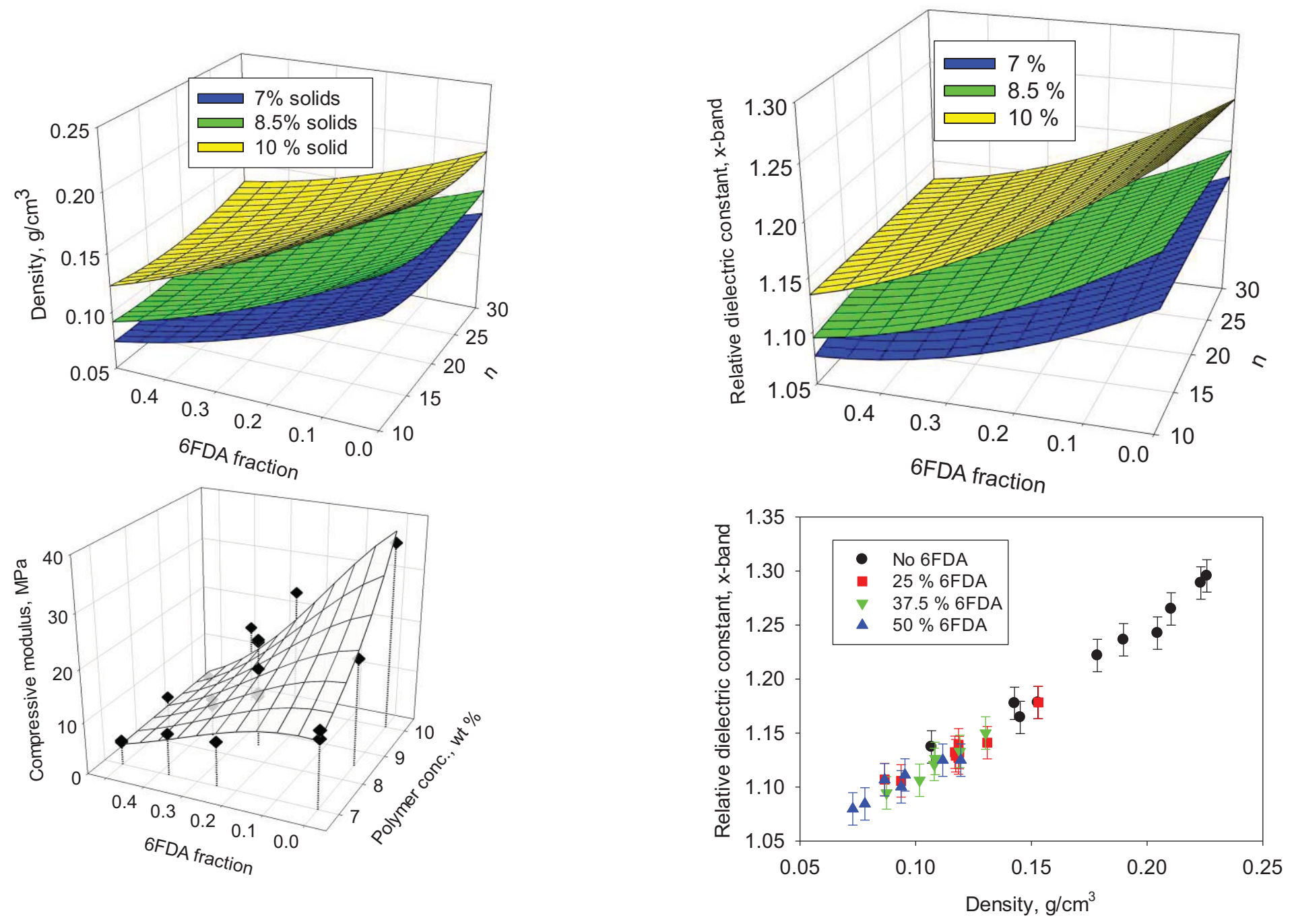
Antenna Prototypes 


\section{Printed Circuit Patch Antennas}

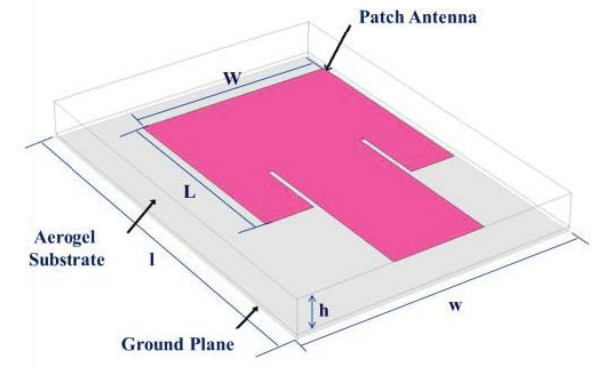

(a)

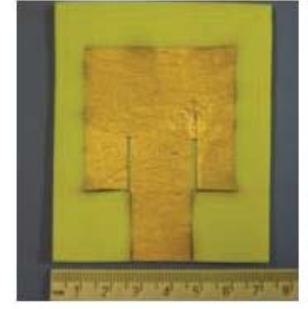

(b)

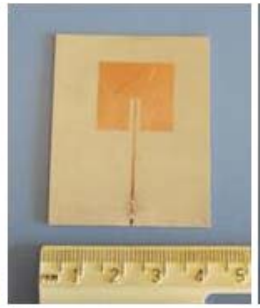

(c)

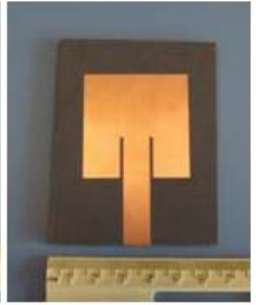

(d)

Schematic of patch antenna (a) and photographs of patch antenna on PI aerogel substrate (b), Rogers Duroid ${ }^{\circledR} 6010\left(\varepsilon_{\mathrm{r}}=10.2\right)(\mathrm{c})$; and Rogers Duroid ${ }^{\circledR}$ $5880\left(\varepsilon_{\mathrm{r}}=2.2\right)(\mathrm{d})$.

\section{Comparison of Duroid and aerogel patch}

\begin{tabular}{|c|c|c|c|c|c|}
\hline Substrate & $\begin{array}{c}\text { Dielectric } \\
\text { constant }\end{array}$ & $\begin{array}{c}\text { 3 dB Bandwidth }(\mathrm{MHz}) \\
\text { simulated/measured }\end{array}$ & $\begin{array}{c}\mathbf{1 0} \text { dB Bandwidth }(\mathbf{M H z}) \\
\text { simulated/measured }\end{array}$ & $\begin{array}{c}\text { Dimensions } \\
\mathbf{1} / \mathbf{w} / \mathbf{h}(\mathbf{c m})\end{array}$ & $\begin{array}{c}\text { Mass } \\
\mathbf{( g})\end{array}$ \\
\hline Duroid 6010 & 10.2 & $16.8 / 22.1$ & $5.6 /--$ & $5.56 / 3.98 / 0.61$ & 4.30 \\
\hline Duroid 5880 & 2.2 & $159 / 192$ & $50.3 / 57.4$ & $8.31 / 6.52 / 0.32$ & 36.45 \\
\hline PI aerogel & 1.16 & $218 / 255$ & $62.1 / 71.2$ & $8.82 / 6.88 / 0.41$ & 4.18 \\
\hline
\end{tabular}

Simulated (sim) and experimentally measured (exp) bandwidths at $3 \mathrm{~dB}$ and $10 \mathrm{~dB}$ for all three substrates.

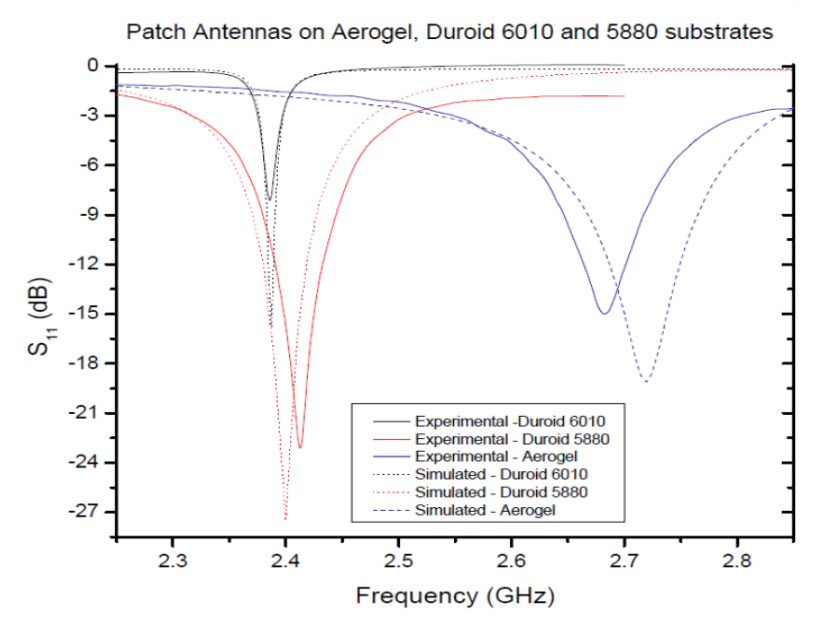

Experimental and simulated $S_{11}$ vs frequency for patch antennas fabricated from $\mathrm{PI}$ aerogel and Rogers Duroid ${ }^{\circledR} 6010$ and 5880.

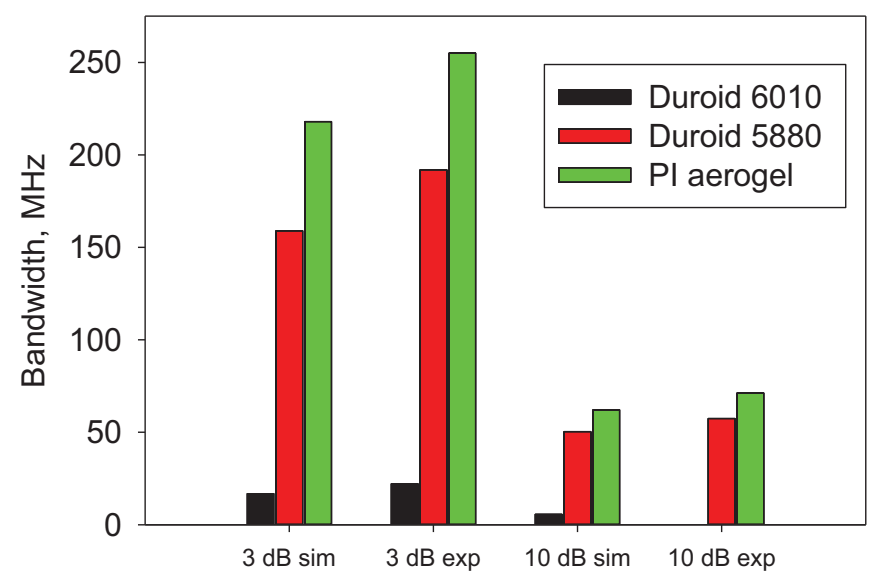

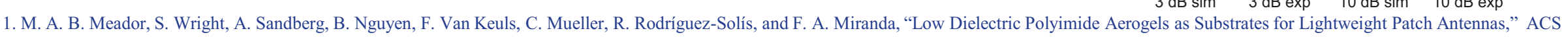
Appl. Mater. Interfaces, 4, 6346-6353, 2012.

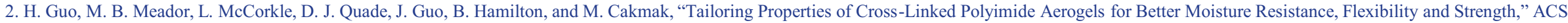
Appl. Mater. Interfaces, 4 (10), 5422-5429, $2012 .$.

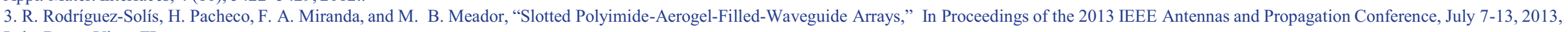
Lake Buena Vista, FL. 


\section{Antenna Gain Measurements}

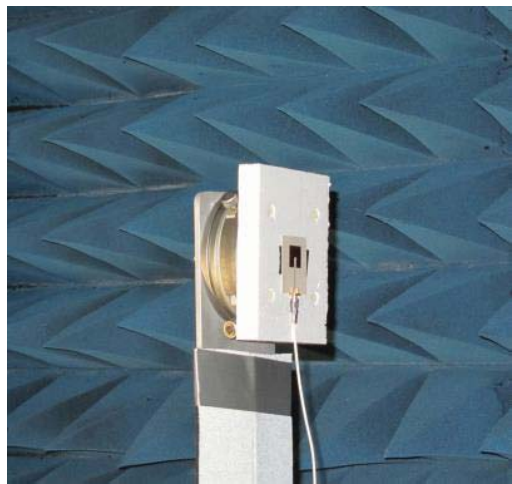

Duroid 6010

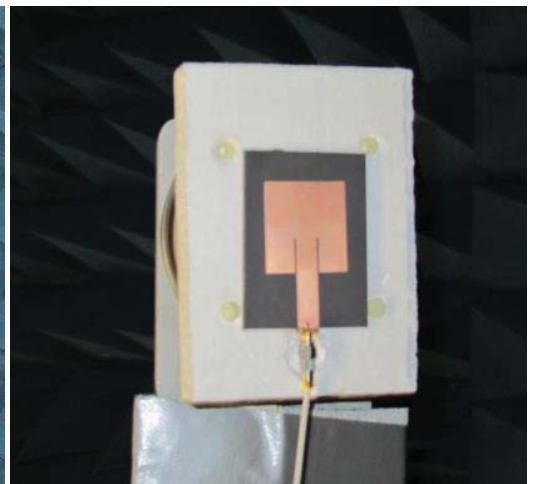

Duroid 5880

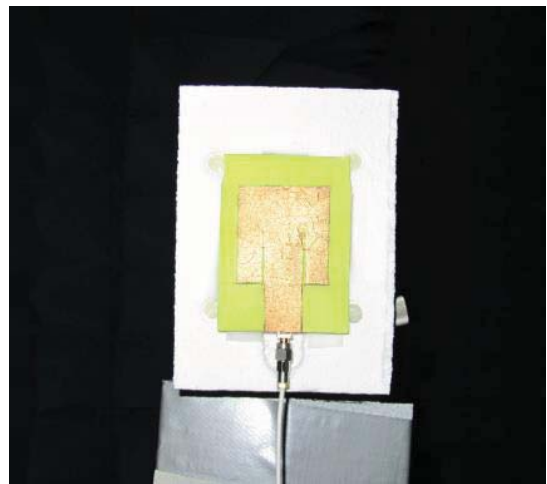

Polyimide Aerogel 1

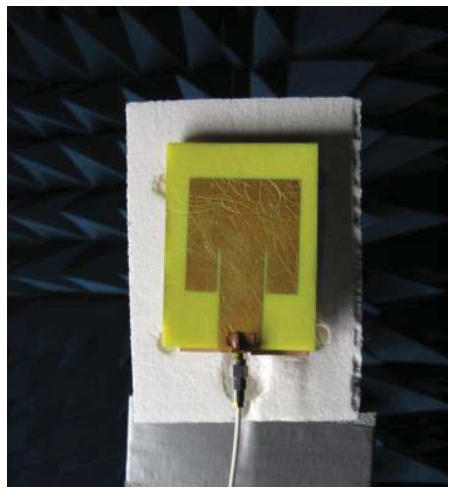

Polyimide Aerogel 2
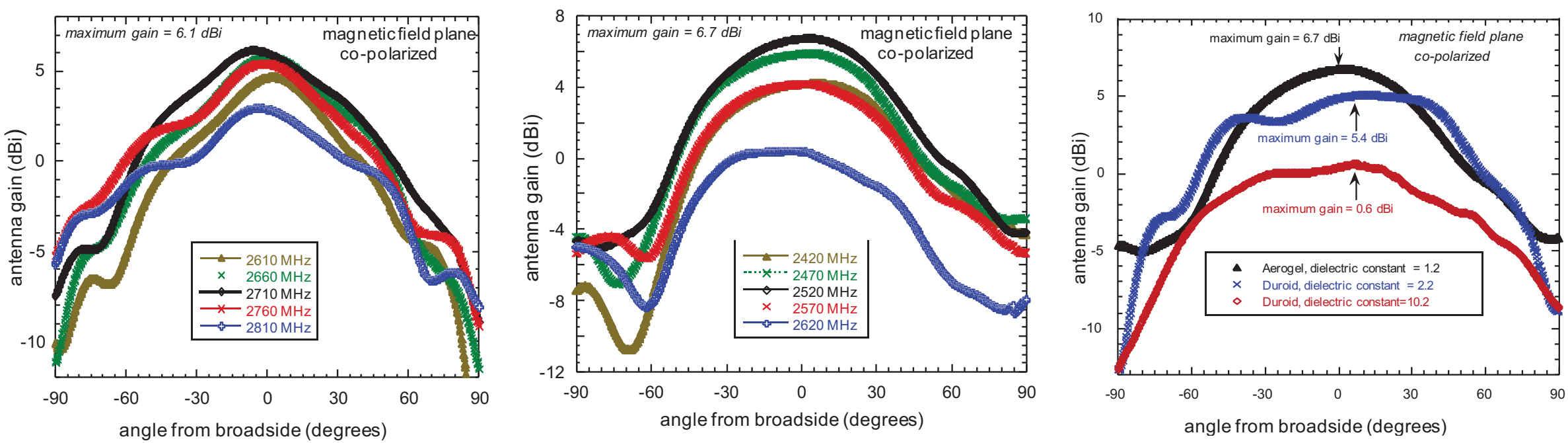

Antenna Gain vs. scan angle from broadside for: (a) Polyimide aerogel antenna \# 1, (b) Polyimide aerogel antenna \# 2, (c) Polyimide aerogel antenna \#2, antenna in Duroid $5880\left(\varepsilon_{\mathrm{r}}=2.2\right)$ and antenna in Duroid $6010\left(\varepsilon_{\mathrm{r}}=10.2\right)$. 


\section{Slot-coupled $5 \mathrm{GHz}$ patch antennas}

- Slot coupled design offers multiple benefits:

- Beam-shaping elements (phase shifters, attenuators) easily inserted into feed network

- Amplifiers (low-noise for receive, high gain for transmit) readily integrated into feed network

- These components are essential for electronically steerable, adaptively controlled antennas

- RF energy coupled from feed network to radiating elements via aperture in ground plane

- Low dielectric of aerogel enables thicker substrate layer for high gain, high bandwidth radiating elements

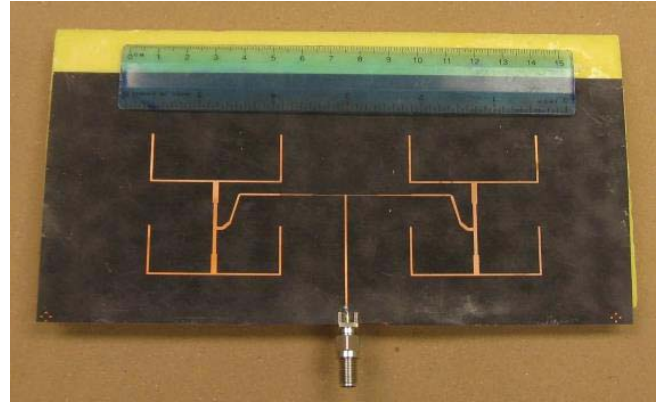

$4 \times 2$ array feed (bottom layer)

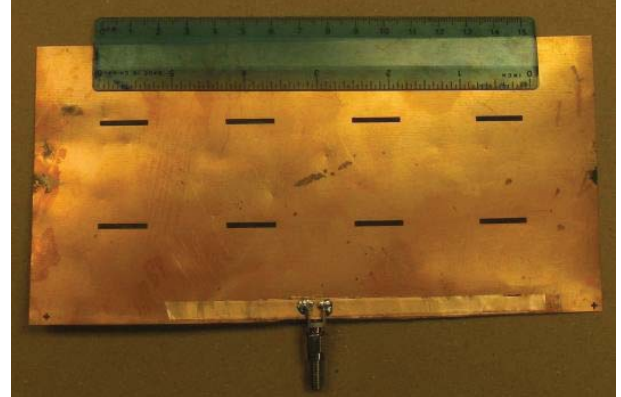

Slotted ground plane (middle layer)

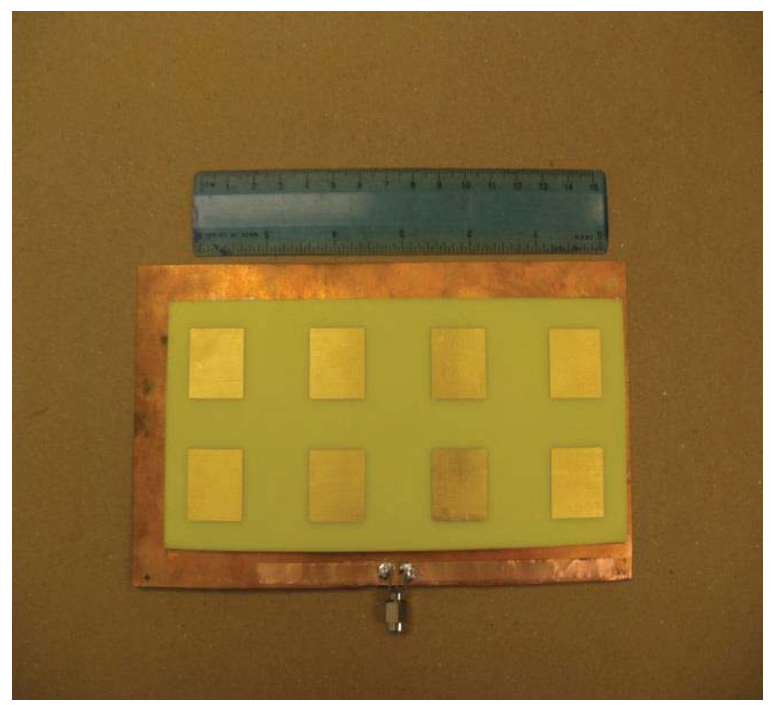

Fully assembled $4 \times 2$ array. Aperture size is $15.3 \mathrm{~cm} \times 5.8 \mathrm{~cm}$

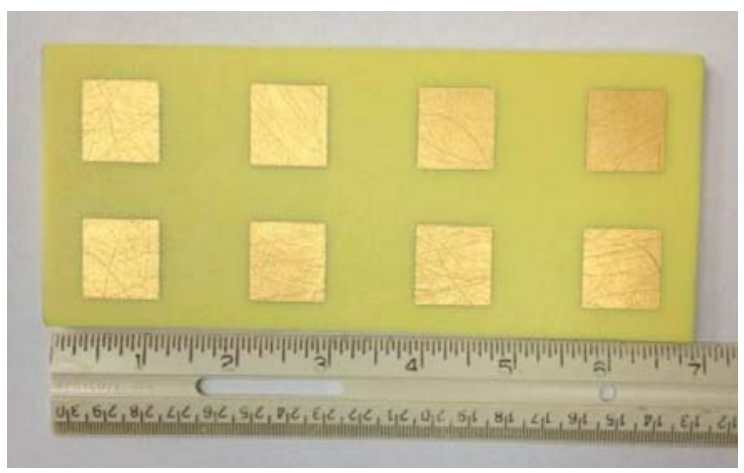

$4 \times 2$ array of radiators on 3 $\mathrm{mm}$ thick PI Aerogel (top layer) 


\section{Slot coupled $2 \times 4$ array antennas}
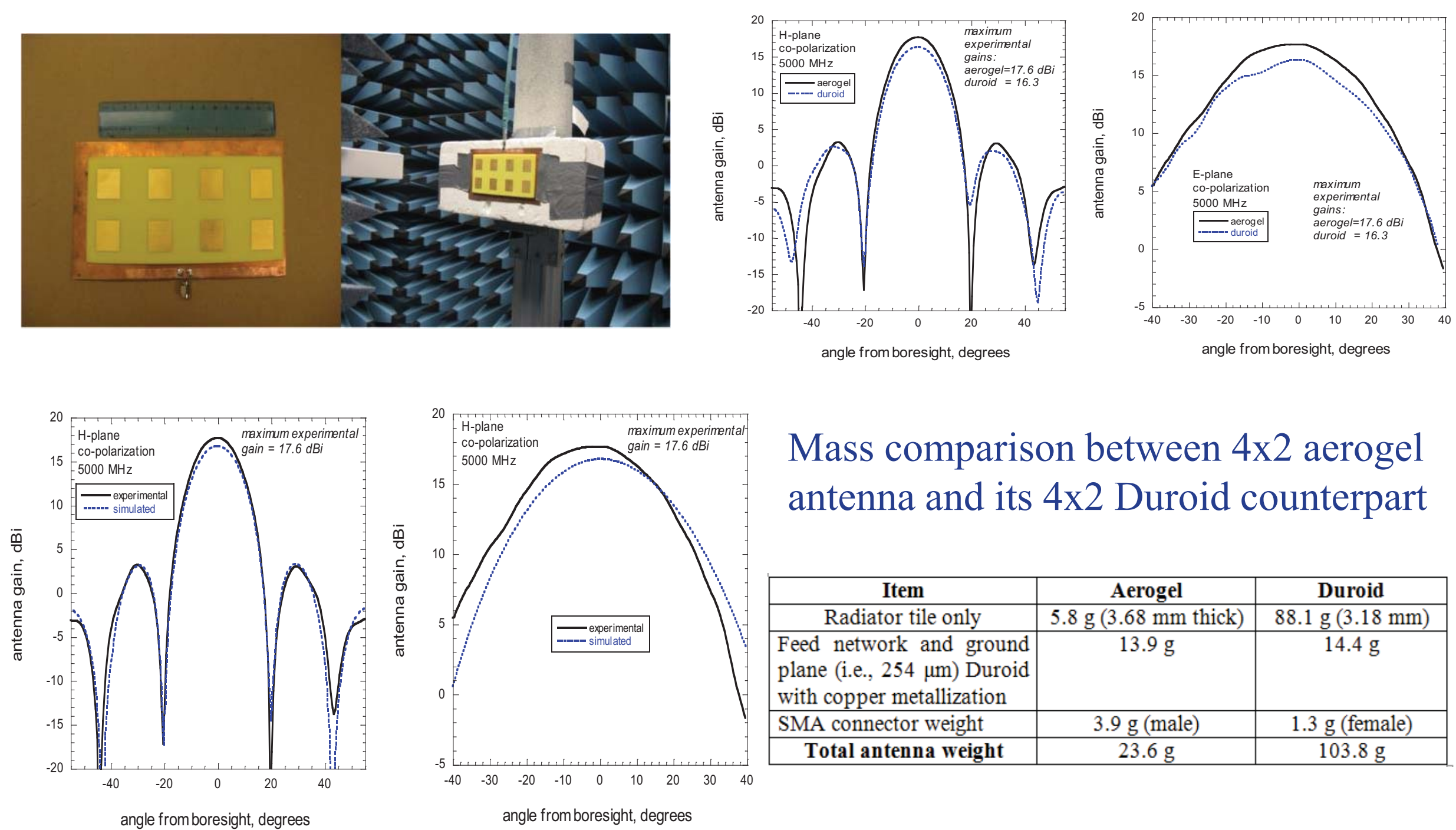

Mass comparison between $4 \times 2$ aerogel antenna and its 4x2 Duroid counterpart

\begin{tabular}{|c|c|c|}
\hline Item & Aerogel & Duroid \\
\hline Radiator tile only & $5.8 \mathrm{~g}(3.68 \mathrm{~mm}$ thick $)$ & $88.1 \mathrm{~g}(3.18 \mathrm{~mm})$ \\
\hline $\begin{array}{l}\text { Feed network and ground } \\
\text { plane (i.e., } 254 \mu \mathrm{m}) \text { Duroid } \\
\text { with copper metallization }\end{array}$ & $13.9 \mathrm{~g}$ & $14.4 \mathrm{~g}$ \\
\hline SMA connector weight & $3.9 \mathrm{~g}$ (male) & $1.3 \mathrm{~g}($ female $)$ \\
\hline Total antenna weight & $23.6 \mathrm{~g}$ & $103.8 \mathrm{~g}$ \\
\hline
\end{tabular}

angle from boresight, degrees

angle from boresight, degrees 


\section{2 element Aerogel Antenna Array}

$>$ Testing of 32 element array

$>$ Shows scalability of aerogel antenna performance. Tile approach used to increase physical aperture size and increase gain

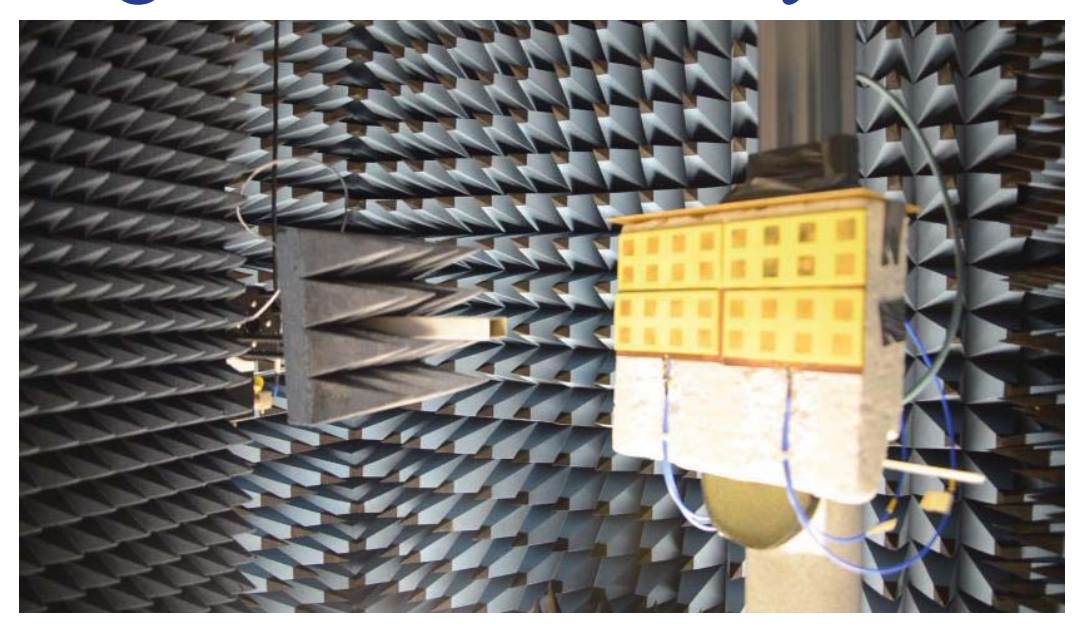

Gain vs. angle from boresight (Simulated and experimental)

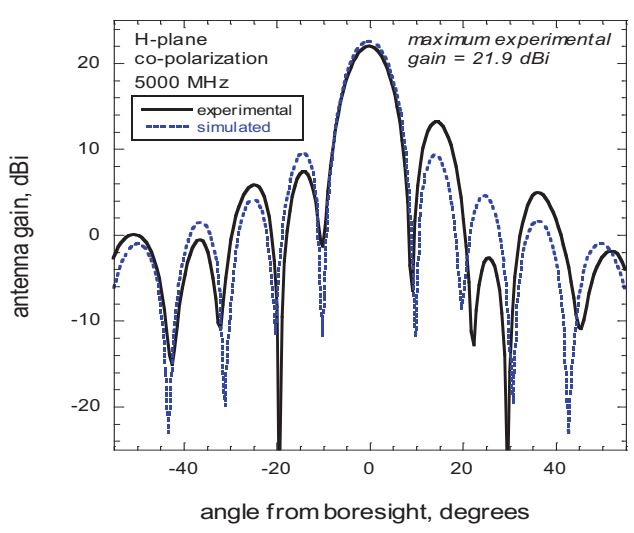

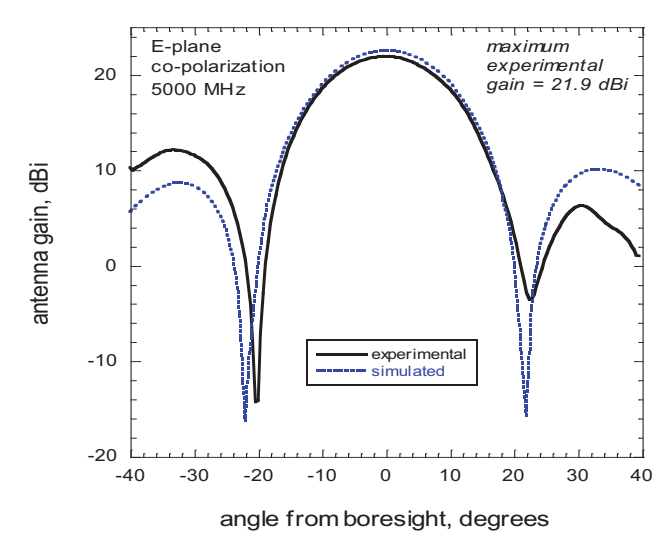

angle from boresight, degrees
Comparative Antenna Grain and Aperture Efficiency

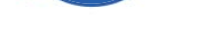




\section{Error Vector Magnitude Measurements}




\section{Error Vector Magnitude (EVM) Measurements}

$>$ Based on the performance of the aerogel antennas a line-of-sight (LOS) experiment was performed to investigate the suitability of these antennas to support digital communication links with typically used modulation schemes.

$>$ An EVM measurement experiment was implemented using two identical 4x2 aerogel arrays, one as transmit $(\mathrm{Tx})$ and the other as receive $(\mathrm{Rx})$ antennas, 8.5 meter apart. The gain of the Tx and $\mathrm{Rx}$ antennas was $15.6 \mathrm{dBi}$.

$>$ Tests were performed at 4600, 5000 and $5400 \mathrm{MHz}$.

$>$ An Agilent 8267D vector signal generator (VSG) was used in the Tx end and an Agilent E4407B spectrum analyzer (SA) with option 229 modulation analysis personality was connected to the Rx antenna at the receiver end of the test set up.

$>$ A symbol rate of 7 Megasamples per second (Msps) was used, which with a spectral efficiency of $2 \mathrm{bits} / \mathrm{symbol}$ resulted in a data rate of $14 \mathrm{Mbps}$. The resolution bandwidth of the SA is set to $10 \mathrm{MHz}$ to enable this data rate.

$>$ The high noise figure of the SA ( $\sim 26 \mathrm{~dB})$, combined with the wide resolution bandwidth, introduced a high noise floor which masked the capability of the Rx antenna to detect low signal power levels. 


\section{Verification of antenna performance in a terrestrial link}

$>$ Line-of-sight experiment performed to investigate suitability of aerogel antennas to support digital communication links with typical modulation schemes (e.g., QPSK and $\pi / 4$ QPSK)

$>$ Error Vector Magnitude (EVM) measurement

- Measure of how far experimental values deviate from reference values

- Two identical 4 x 2 aerogel arrays used as transmit (Tx) and receive $(\mathrm{Rx})$ antennas.

- Separation of $8.5 \mathrm{~m}$ to satisfy $2 \mathrm{D}^{2} / \lambda$ far field criteria

- $\mathrm{D}$ is the maximum antenna aperture dimension

- $\lambda$ is the wavelength corresponding to the frequency of the array

- In our case, $\mathrm{D}=16.1 \mathrm{~cm}, \lambda=6.0 \mathrm{~cm}$, and $2 \mathrm{D}^{2} / \lambda=0.864$ meters
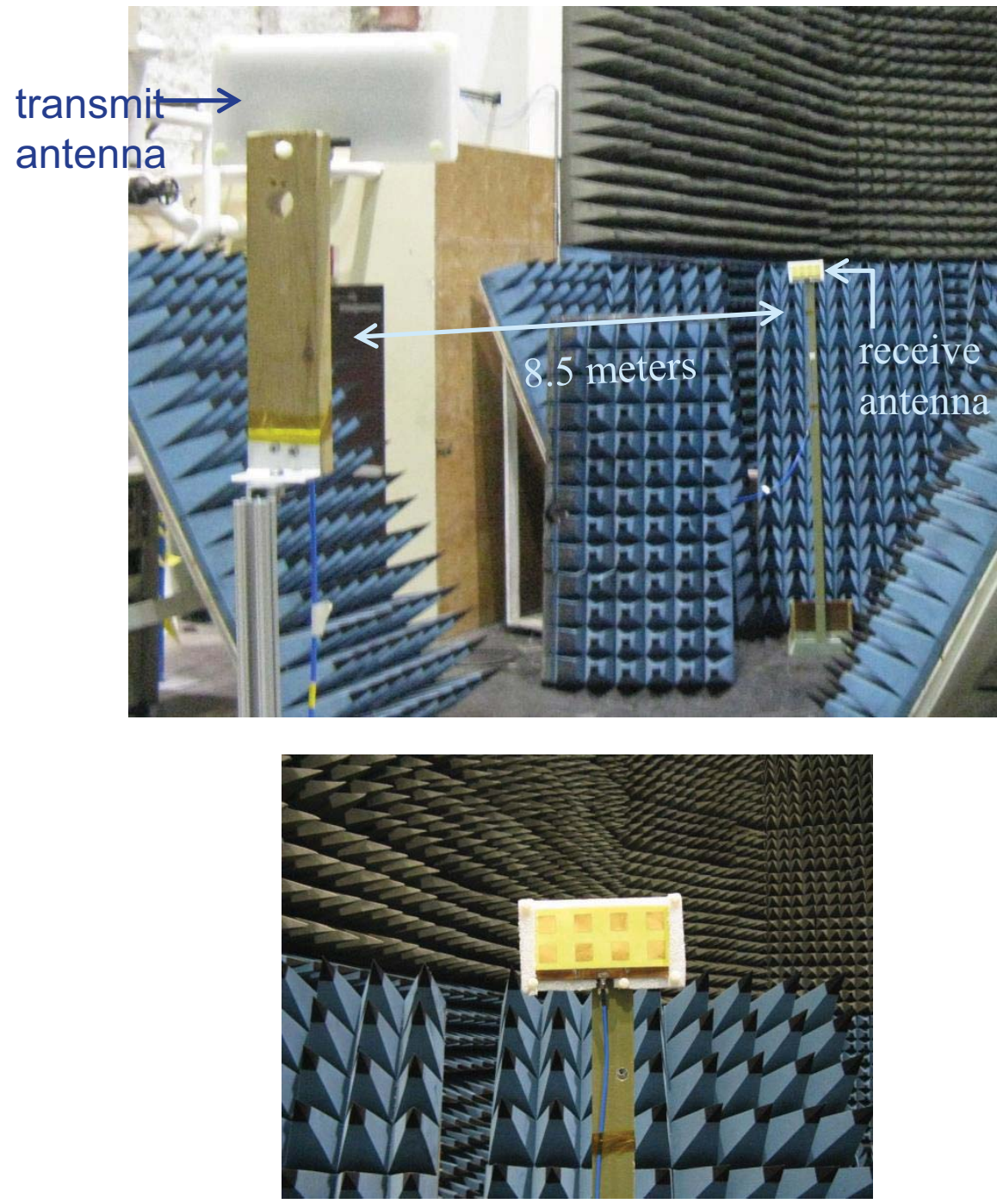


\section{Error Vector Magnitude (EVM) Measurements}

The following parameters are used in the experiment:

* Transmit power $=-23 \mathrm{dBm}, 20 \mathrm{dBm}, 0 \mathrm{dBm}$, and $20 \mathrm{dBm}$

* Tx and Rx antenna gain $=15.6 \mathrm{dBi}$

* Link distance: 8.5 meters

* Frequency $=5.0 \mathrm{GHz}$

* Data rate $=14$ Mbps

* Modulation schemes= quadrature phase shift keying (QPSK) and $\pi / 4$ differential QPSK ( $\pi / 4$ DQPSK). 
Constellation plots for QPSK and $\pi / 4$ DQPSK modulation schemes at different input powers

(No amplifier between the Rx antenna and the SA)

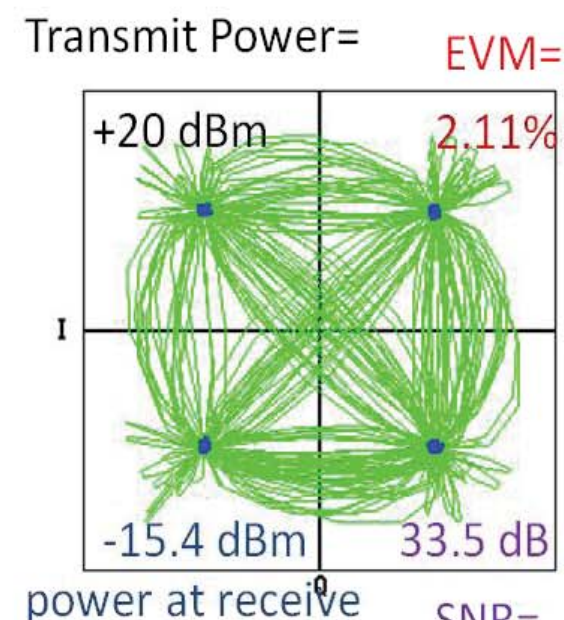
pNR= antenna aperture=
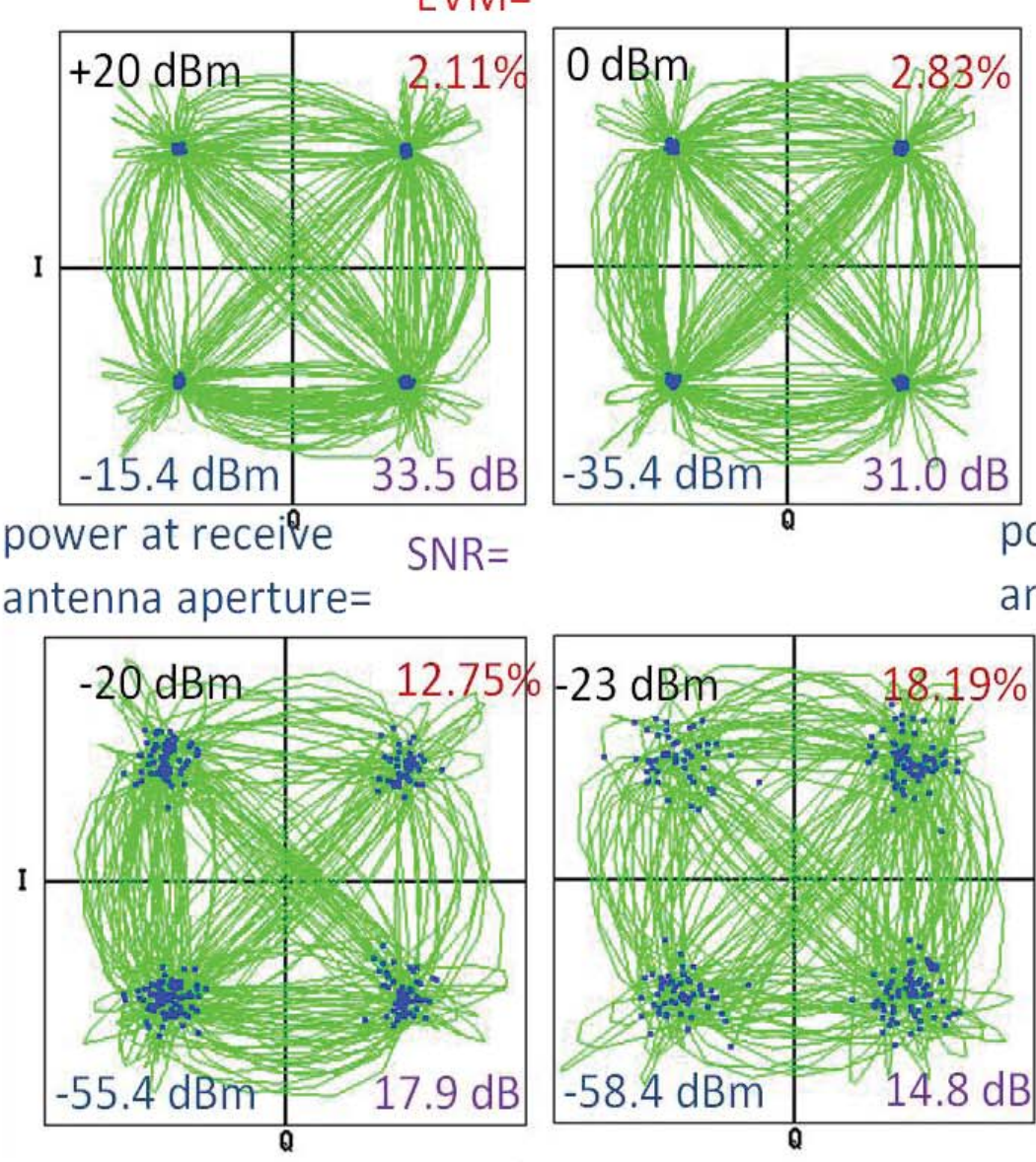

Transmit Power $=\quad \mathrm{EVM}=$
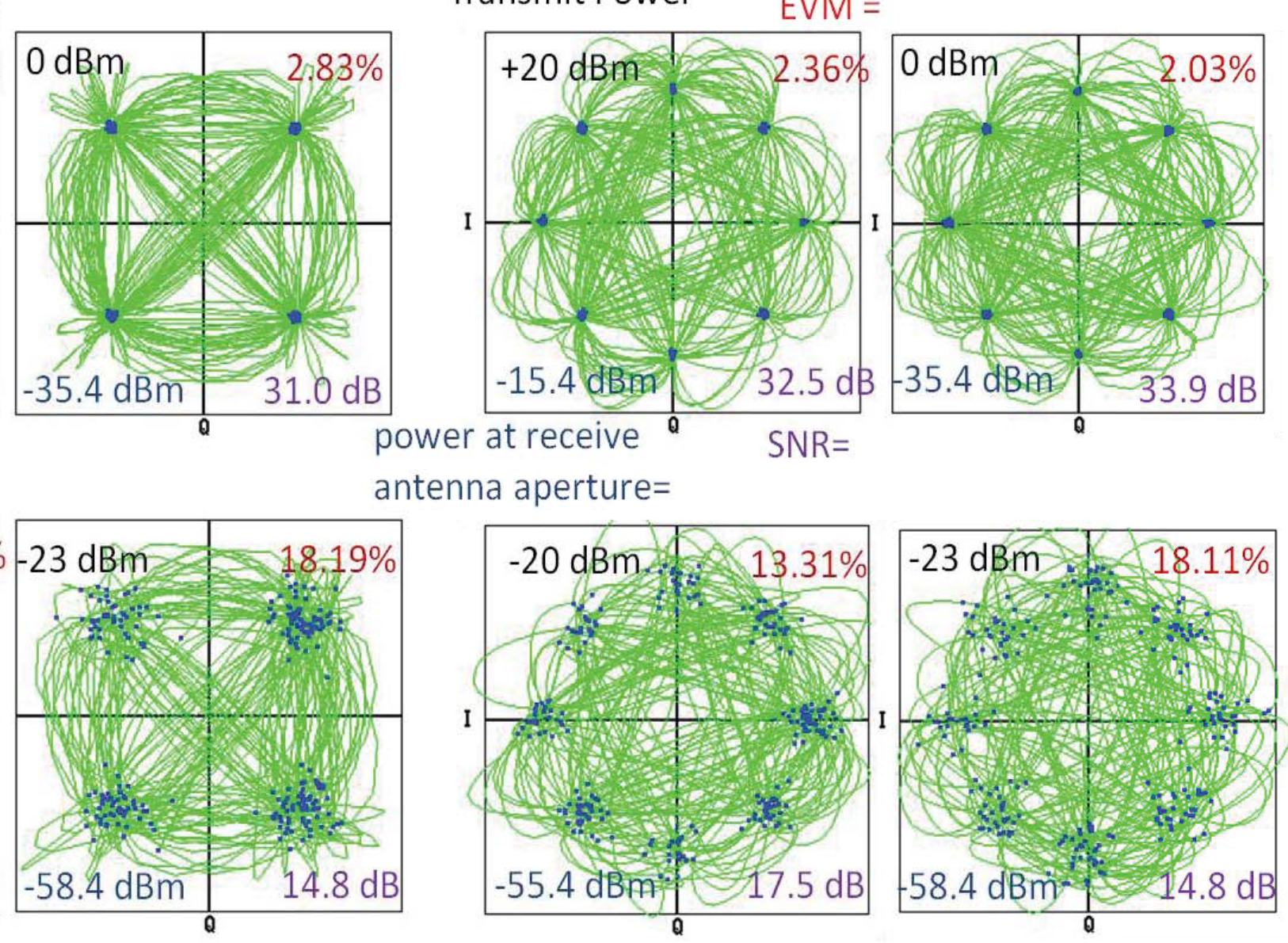

Aerogel Phased Array Antennas EVM Test using QPSK (left) and ( $\pi / 4$ DQPSK) Modulation Schemes. 


\section{Error Vector Magnitude (EVM) Measurements}

$>$ As expected, for both modulation schemes the EVM increases and the SNR decreases with decreasing Tx power.

$>$ In other words, the increased spreading of the constellation points with decreasing power is an indication of the degradation of the link quality.

$>$ When an Avantek AWT-6053 amplifier (small signal gain of $27 \mathrm{~dB}$ and a noise figure of $4 \mathrm{~dB}$ ) is inserted between the SA and the $\mathrm{Rx}$ antenna, the aerogel antennas are able to support a link even when the power levels at the receive antenna aperture are as low as -83.4 $\mathrm{dBm}$. 
Constellation plots for QPSK and $\pi / 4$ DQPSK modulation schemes at different input powers

(With amplifier between the Rx antenna and the SA)

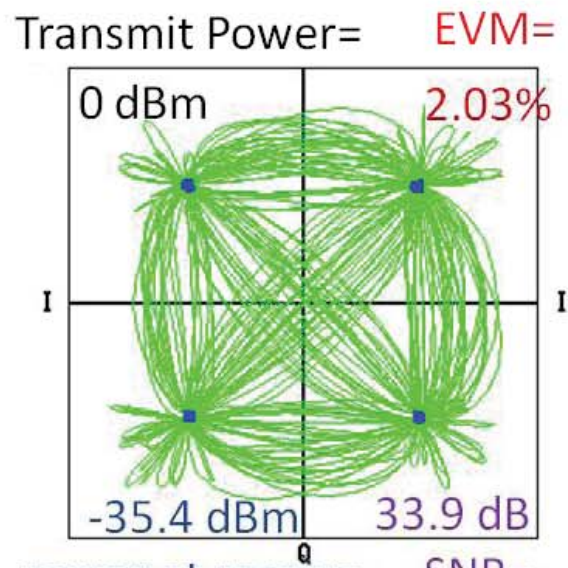
power at receive $\mathrm{SNR}=$

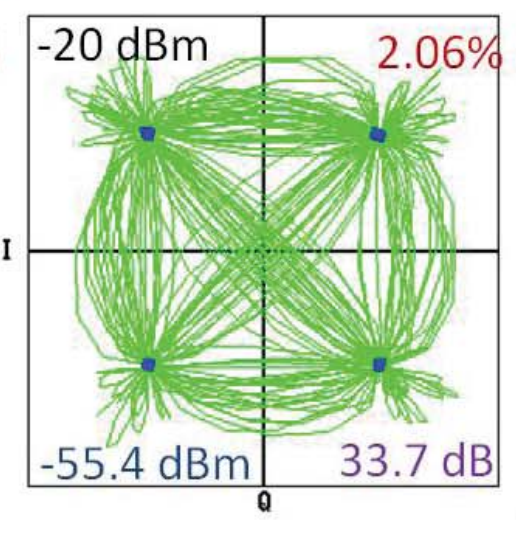

antenna aperture $=$
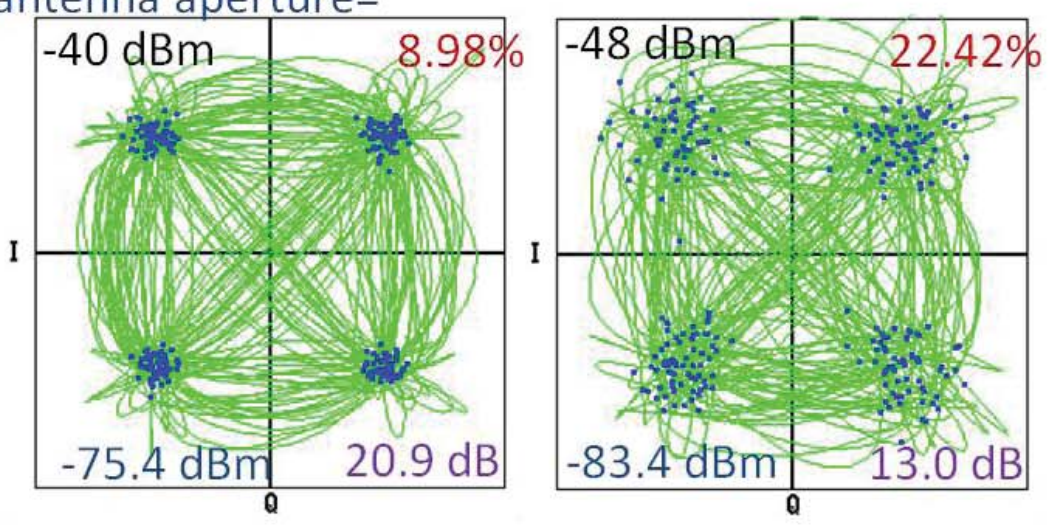

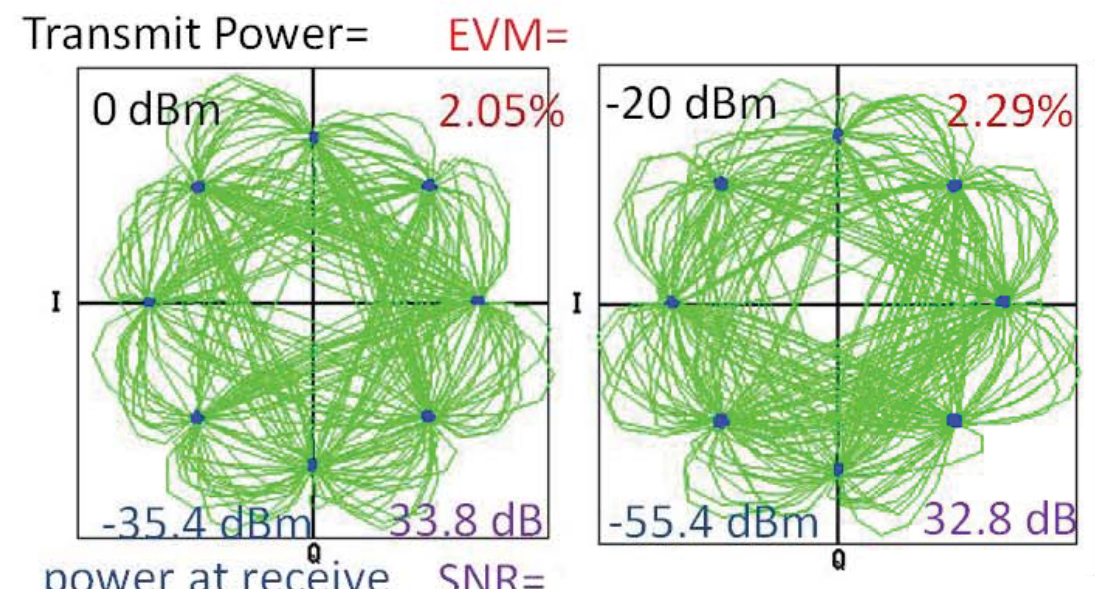
power at recêive SNR=

antenna aperture $=$
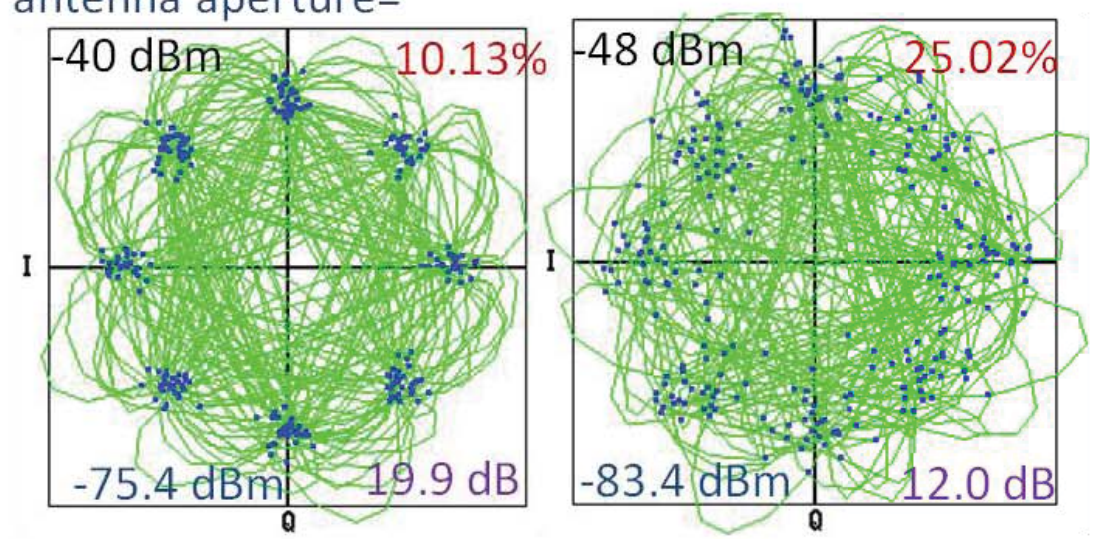

Aerogel Phased Array Antennas EVM Test using QPSK (left) and ( $\pi / 4$ DQPSK) modulation schemes 
EVM and SNR versus transmit power at $5.0 \mathrm{GHz}$ (top) and $4.6 \mathrm{GHz}$ (bottom) for the two modulation schemes discussed in this work.
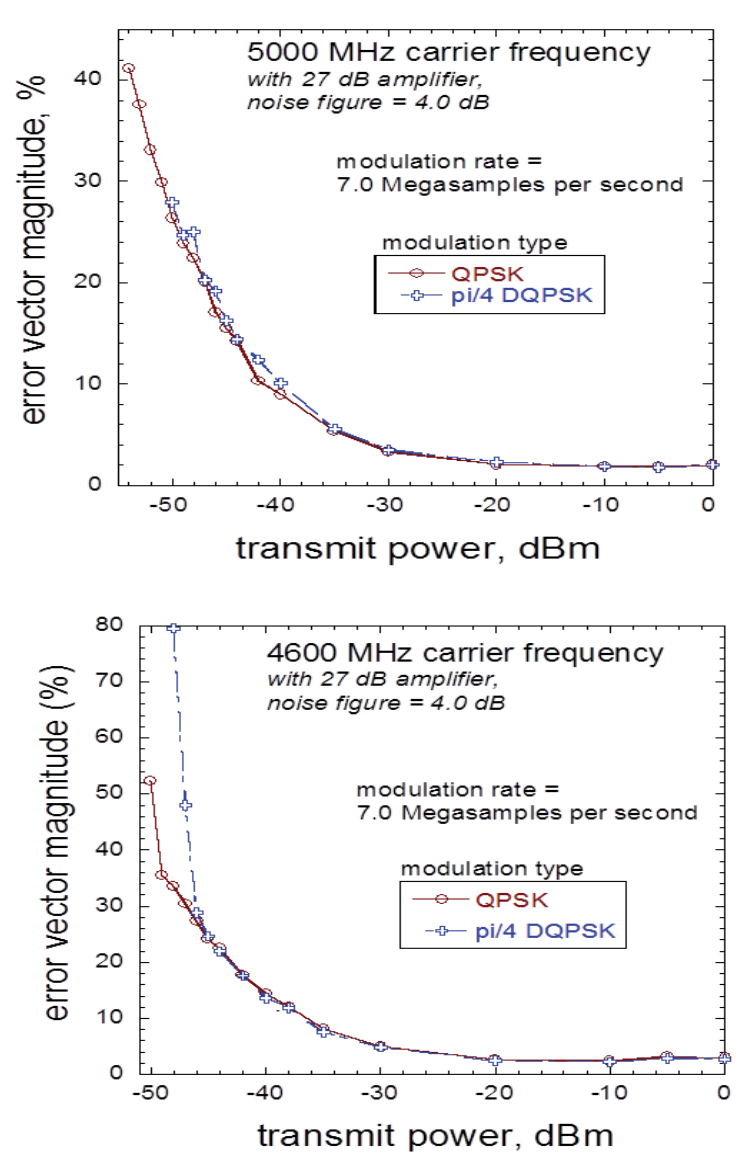
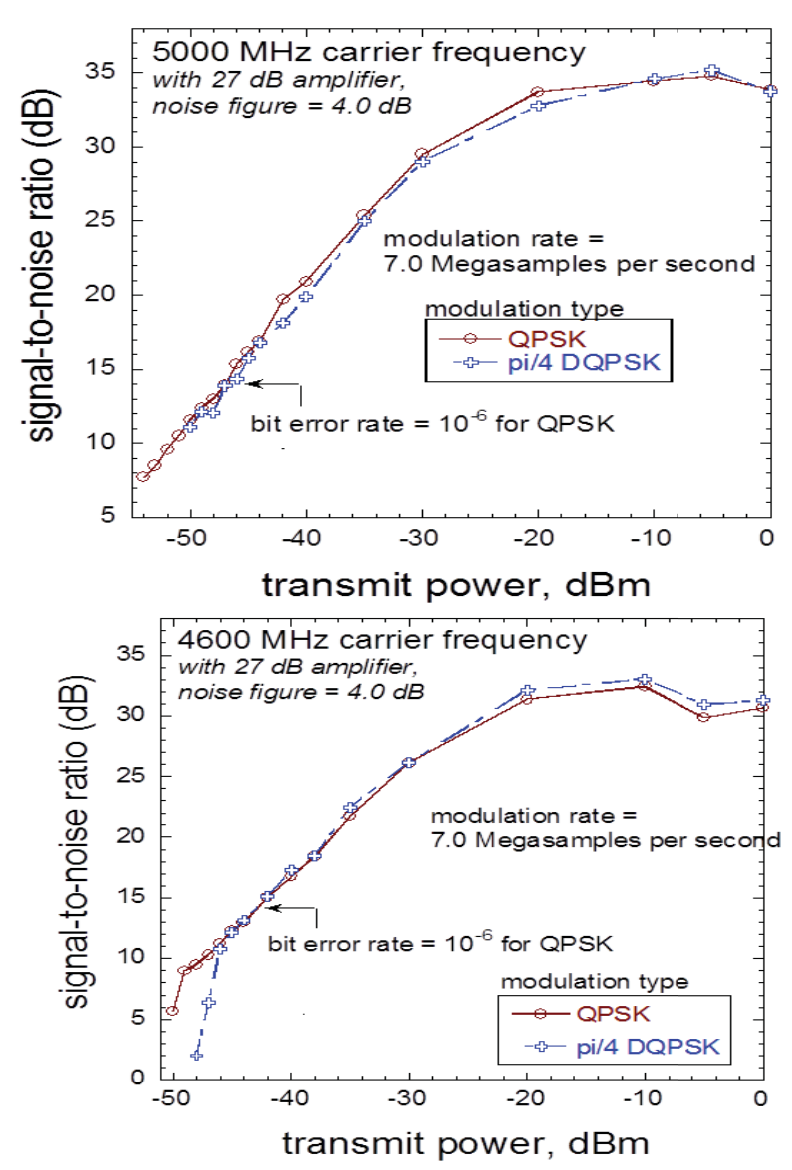

Note that even at 4.6 $\mathrm{GHz}$, the antennas are able to maintain a communication link with only $\sim 2 \mathrm{~dB}$ loss relative to the antenna performance at $5.0 \mathrm{GHz}$.

Similar results were also observed at $5.4 \mathrm{GHz}$. 


\section{Achievable data rates with different link scenarios}

Aerogel transmit and receive antennas (2x4 element phased arrays, $15.6 \mathrm{dBi}$ gain) at $5000 \mathrm{MHz}$, communications link from directly overhead to $10^{\circ}$ from horizon

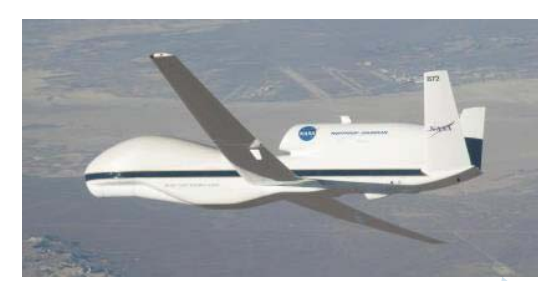

unmanned aerial vehicle $(70,000 \mathrm{ft})$

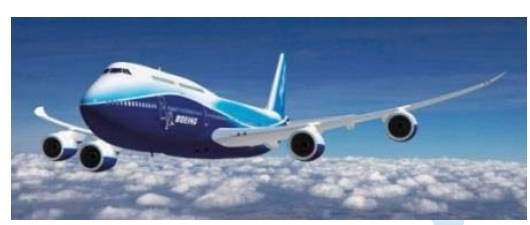

commercial airplane $(30,000 \mathrm{ft})$

ground terminal

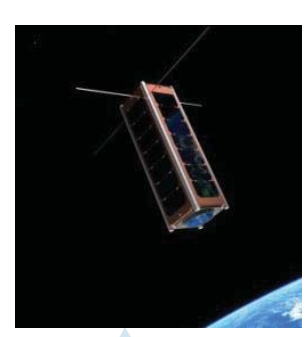

cubesat $(350 \mathrm{~km})$

- Data rates in table below are based on the following conditions:

- Transmit power = 2 watt

- Gain for both transmit and receive antennas $=15.6 \mathrm{dBi}$

- Receiver noise figure $=4 \mathrm{~dB}$

- QPSK modulation

- Free space losses based on link distance when vehicle located 10 degrees elevation above the horizon

- Signal-to-noise $\geq 14 \mathrm{~dB}$, bit error rate $\leq 10^{-6}$

- No coding gain

- Receiver implementation losses $=3 \mathrm{~dB}$

-When vehicle is directly overhead (see figures) free space losses are reduced by $7.5 \mathrm{~dB}$

- Allowable data rates are 5.7 times higher than when the vehicle is 10 degrees elevation above the horizon

\begin{tabular}{|l|c|c|}
\hline \multicolumn{1}{|c|}{ Link } & $\begin{array}{c}\text { Maximum link } \\
\text { distance }(\mathbf{k m})\end{array}$ & $\begin{array}{c}\text { Data rate } \\
\text { supported }\end{array}$ \\
\hline Cubesat-to-ground & 2000 & $38 \mathrm{~kb} / \mathrm{s}$ \\
\hline UAV-to-ground & 120 & $10 \mathrm{Mb} / \mathrm{s}$ \\
\hline Commercial airplane-to-ground & 51 & $58 \mathrm{Mb} / \mathrm{s}$ \\
\hline
\end{tabular}




\section{Conclusions}

$>$ The suitability of Polyimide aerogel antennas for supporting digital communication links with traditionally used modulation schemes was demonstrated through error vector magnitude measurements has been discussed in this paper.

$>$ The EVM data indicate that $2 \times 4$ aerogel polyimide arrays can support digital communications links under commonly used modulation schemes for aerospace communication applications.

$>$ Besides communications these antennas could be applicable to radars mounted in small platforms (i.e., CubeSats and UAV's) where their bandwidth, Gain, and weight could be used advantageously. 


\section{References}

1. M. A. B. Meador, S. Wright, A. Sandberg, B. Nguyen, F. Van Keuls, C. Mueller, R. Rodríguez-Solís, and F. A. Miranda, "Low Dielectric Polyimide Aerogels as Substrates for Lightweight Patch Antennas," ACS Appl. Mater. Interfaces, 4, 6346-6353, 2012.

2. H. Guo, M. B. Meador, L. McCorkle, D. J. Quade, J. Guo, B. Hamilton, and M. Cakmak, "Tailoring Properties of Cross-Linked Polyimide Aerogels for Better Moisture Resistance, Flexibility and Strength," ACS Appl. Mater. Interfaces, 4 (10), 5422-5429, 2012.

3. R. Rodríguez-Solís, H. Pacheco, F. A. Miranda, and M. B. Meador, "Slotted Polyimide-Aerogel-Filled-Waveguide Arrays," In Proceedings of the 2013 IEEE Antennas and Propagation Conference, July 7-13, 2013, Lake Buena Vista, FL. 


\section{Acknowledgements}

- Aerogel fabrication

- Ms. Sarah Wright (Cal Tech intern)

- Liz Barrios, Anna Sandberg, Emily MacMillon (USRP)

- Dr. Baochau N. Nguyen (RXD/OAI)

- RF testing, simulations, antenna characterization

- Dr. Fred W. Van Keuls (RHA/Vantage Partners, Inc.)

- Ms. Elizabeth McQuaid (CS-FTF)

- Mr. Nicholas Varaljay (CS-FTF)

- Rafael Rodríguez-Solís, (University of Puerto Rico-Mayagüez)

- $\quad$ Funding

- NASA ARMD Seedling Proposal Program 